(c) American Dairy Science Association, 2006.

\title{
A Method to Define Breeding Goals for Sustainable Dairy Cattle Production
}

\author{
H. M. Nielsen, ${ }^{\star 1}$ L. G. Christensen,† and J. Ødegård* \\ *Department of Animal and Aquacultural Sciences, Norwegian University of Life Sciences, PO Box 5003, N-1432 Ås, Norway \\ †Department of Large Animal Sciences, The Royal Veterinary and Agricultural University. Grønnegårdsvej 8, \\ DK-1970 Frederiksberg C, Denmark
}

\section{ABSTRACT}

The objective of this study was to present a method to define breeding goals for sustainable dairy cattle production by adding nonmarket values to market economic values for functional traits in the breeding goal. A nonmarket value can represent the value of improved animal welfare or societal influences for animal production. The nonmarket value for mastitis resistance, conception rate, and stillbirth were derived based on how much farmers or breeding companies were willing to lose in selection response for milk yield to improve functional traits. The desired response for milk yield corresponding to a given percent loss was obtained using desired gain indices. By allowing a 5\% loss in the selection response for milk yield, the nonmarket value was found to be $€ 40.4$ for mastitis resistance, $€ 16.1$ for conception rate, and $€-9.7$ for stillbirth. The nonmarket value increased proportionally with increasing loss in the selection response for milk yield, but the selection response was lower for conception rate than for mastitis resistance because of differences in market economic value and heritability. To increase the response for conception rate, the nonmarket value was also derived for 2 situations, in which the desired responses for milk yield, mastitis resistance, and conception rate were specified. The method can be used to define breeding goals for sustainable production and to increase the response for traits that are at critically low levels. When defining breeding goals for sustainable production, breeding organizations should predict the selection response based on market economic value and add nonmarket value for traits with unacceptable selection responses.

Key words: breeding objective, desired gain index, sustainability

\section{INTRODUCTION}

The concept of sustainability in agricultural production has received increased attention. In addition, con-

Received January 31, 2006.

Accepted April 7, 2006.

${ }^{1}$ Corresponding author: hanne.marie.nielsen@umb.no sumers are interested in products produced in a sustainable way. Therefore, animal breeders should engage in discussions concerning sustainability (Gamborg and Sandøe, 2005). An overall definition of sustainable animal breeding is "the extent to which animal breeding and reproduction, as managed by professional organisations, contribute to the maintenance and good care of animal genetic resources for future generations" (Gamborg and Sandøe, 2005). Key words characterizing sustainable animal breeding are product quality, genetic diversity, efficiency, environment, and animal health and welfare (Code-EFABAR, 2004).

Breeding goals for dairy cattle are currently moving toward including both production and functional traits (Mark, 2004; Miglior et al., 2005). Functional traits, such as mastitis resistance (MR) and rate of stillbirth (SB), are related to animal health and welfare, and thus sustainability. Hence, traits related to sustainability are already considered in dairy cattle breeding, but selection for increased milk yield may nevertheless result in deterioration of functional traits even when functional traits are given some weight in the breeding goal. Deterioration of functional traits because of selection for milk yield arises because many functional traits are unfavorably genetically correlated with production traits and because functional traits often have lower heritability and lower market economic value (EV) than do production traits. A breeding goal resulting in deterioration of functional traits such as health- and welfarerelated traits because of selection for production traits is in conflict with animal welfare (Sandøe et al., 1999), and thus with the term sustainability. Farm animal breeders need to balance genetic improvement in production traits with genetic improvement in functional traits such that functional traits are improved (or the decline is minimized) and overall productivity and efficiency are increased (Gamborg and Sandøe, 2005).

The traditional methods used to derive market EV in the breeding goal-by using profit equations (e.g., Brascamp et al., 1985), with the objective of maximizing the profit of the farmer-do not consider the concept of sustainability. The idea of adding a nonmarket value (NV) to the EV in the breeding goal (Hazel, 1943) was 
introduced by Olesen et al. (2000). An NV is a value that is not reflected in the current market economy or that is only partly transferred via the market. For example, it can be an ethical value of improved animal welfare caused by genetic improvement, which is not reflected in the current market prices of products. By including NV, the value of improved animal welfare and social aspects can be considered when defining the breeding goal (Olesen et al., 2000). However, even though tools for deriving NV have been proposed (Olesen et al., 1999), only a few examples in the literature show how to derive NV for traits in the breeding goal (Kanis et al., 2005, for pigs; Nielsen et al., 2005, for dairy cattle). In the method of Nielsen et al. (2005), NV were derived by quantifying the trade-off between the selection response for milk yield and the selection response for functional traits. However, the method allows for derivation of $\mathrm{NV}$ for only one functional trait at a time. Today, most dairy cattle breeding objectives include milk production and several functional traits (Mark, 2004; Miglior et al., 2005). Hence, there is a need to develop methods to derive NV for multiple functional traits. The objective of this study was to extend the method of Nielsen et al. (2005) to allow for simultaneous derivation of $\mathrm{NV}$ for multiple functional traits using desired gain indices.

\section{MATERIALS AND METHODS}

\section{Breeding Goal, and Genetic and Economic Parameters}

The traits milk yield (MY), MR, conception rate (CR), and SB were included in both the selection index and the breeding goal. Milk yield was weighted only by its $\mathrm{EV}$, whereas all 3 functional traits were weighted by both their EV and their NV (Olesen et al., 2000). The breeding goal or aggregate genotype $(H)$ could then be written as

$$
\begin{aligned}
H=\mathrm{EV}_{\mathrm{MY}} & \times \mathrm{BV}_{\mathrm{MY}}+\left(\mathrm{EV}_{\mathrm{MR}}+\mathrm{NV}_{\mathrm{MR}}\right) \times \mathrm{BV}_{\mathrm{MR}} \\
+ & \left(\mathrm{EV}_{\mathrm{CR}}+\mathrm{NV}_{\mathrm{CR}}\right) \times \mathrm{BV}_{\mathrm{CR}} \\
& +\left(\mathrm{EV}_{\mathrm{SB}}+\mathrm{NV}_{\mathrm{SB}}\right) \times \mathrm{BV}_{\mathrm{SB}}
\end{aligned}
$$

where EV and NV are the market economic value and nonmarket value for MY, MR, CR, and SB. The sum of $\mathrm{EV}$ and $\mathrm{NV}$ is taken as the goal value $(\mathbf{G V})$, and $\mathrm{BV}$ is the genetic value of a selection candidate for each of the 4 traits.

Only the selection paths of progeny-tested sires to breed cows and sires were included. The progeny group per sire was assumed to be 100 daughters, and $5 \%$ of tested bulls were selected (selection intensity, $i=2.06$ ).
Applied genetic correlations, phenotypic correlations (Sørensen, 1999), and economic parameters (Nielsen, 2004) are given in Table 1.

\section{Derivation of NV by Restricting the Selection Response in MY and Functional Traits}

In the study by Nielsen et al. (2005), NV were derived based on how much selection response for MY farmers or breeding companies were willing to lose to improve a given functional trait. Selection response for MY was predicted for increasing levels of $\mathrm{GV}(\mathrm{EV}+\mathrm{NV})$ for a single functional trait. Percent loss in selection response for MY (PCTLOSS) was then predicted as a selection response for MY when the breeding goal contained EV for all traits and a nonzero NV for a single functional trait, in a percentage of selection response for MY aiming at a breeding goal with EV alone for all traits. Next, the selection response and corresponding GV for the functional trait was quantified for each level of PCTLOSS in MY. Finally, the NV for each PCTLOSS was derived as the difference between the GV and the EV. However, the disadvantage with the method described by Nielsen et al. (2005) is that NV can be derived for only one trait at a time. For example, if one is willing to lose 5 PCTLOSS in MY, one has to choose whether the 5 PCTLOSS should be used to improve MR, CR, or $\mathrm{SB}$, but it is not possible to distribute the loss in response in MY to all 3 traits. In the current study, the method of Nielsen et al. (2005) was developed further. The PCTLOSS in selection response for MY was restricted to a given value to allow for simultaneous derivation of $\mathrm{NV}$ for multiple traits. Nonmarket value was derived using 3 different desired gain indices:

- MY restricted to a given PCTLOSS (RMY index)

- MY restricted to a given PCTLOSS and MR restricted to 0 response (RMR index)

- Response in MY restricted to a given PCTLOSS and relative response between MR and CR restricted (RREL index)

\section{Desired Gain Index Theory}

In selection index theory (Hazel, 1943), the maximal selection response is obtained when

$$
\mathbf{P b}=\mathbf{G v}
$$

where $\mathbf{P}$ is a phenotypic (co)variance matrix, $\mathbf{G}$ is a genetic co(variance) matrix, $\mathbf{v}$ is a vector of $\mathrm{EV}$, and $\mathbf{b}$ is a vector of selection index weights, which can be derived as 
Table 1. Applied genetic and phenotypic parameters and market economic values (EV) for milk yield, mastitis resistance, conception rate, and stillbirth ${ }^{1}$

\begin{tabular}{|c|c|c|c|c|c|c|}
\hline Trait $^{1}$ & $\begin{array}{l}\text { Milk } \\
\text { yield }\end{array}$ & $\begin{array}{l}\text { Mastitis } \\
\text { resistance }\end{array}$ & $\begin{array}{l}\text { Conception } \\
\text { rate }\end{array}$ & Stillbirth & $\begin{array}{l}\text { Phenotypic } \\
\text { standard } \\
\text { deviation }\end{array}$ & $\begin{array}{l}\text { EV, } \\
€^{2} \text { /unit }\end{array}$ \\
\hline Milk yield & 0.28 & -0.35 & -0.35 & 0 & 530 & 0.28 \\
\hline Mastitis resistance & 0.03 & 0.04 & 0.20 & 0 & 1 & 163 \\
\hline Conception rate & -0.10 & 0 & 0.03 & 0 & 33 & 1.98 \\
\hline Stillbirth & 0 & 0 & 0 & 0.04 & 24 & -39 \\
\hline
\end{tabular}

${ }^{1}$ Heritabilities (diagonal), genetic correlations (above diagonal) and phenotypic correlations (below diagonal). Units: milk yield $=\mathrm{kg} / \mathrm{cow}$ per yr; mastitis resistance $=$ incidence/cow per yr; conception rate and stillbirth $=\% /$ cow per yr.

${ }^{2} € 1=\$ 1.17$.

$$
\mathbf{b}=\mathbf{P}^{-1} \mathbf{G v} \text {. }
$$

Selection response or genetic superiority of selected sires for a given trait $j$ is predicted as

$$
\Delta \mathbf{G}_{\mathbf{j}}=\frac{\mathbf{G}_{\mathbf{j}}^{\prime} \mathbf{b}}{\sigma_{\mathbf{I}}} \mathbf{i}
$$

and thus

$$
\mathbf{G}_{\mathbf{j}}^{\prime} \mathbf{b}=\frac{\Delta \mathbf{G}_{\mathbf{j}}}{\mathbf{i}} \sigma_{\mathbf{I}}
$$

where $\left(G_{j}^{\prime} b\right)$ is the covariance between index $(I)$ and the additive genotype for trait $j, \sigma_{\mathrm{I}}$ is the standard deviation of the index, and $\mathbf{i}$ is the selection intensity.

In a desired gain index, selection response for one or more traits is restricted to a specified desired level. A desired gain index can be derived from the traditional selection index (Equation 1) by adding a row and column to $\mathbf{P}$ consisting of the $j$ th column of $\mathbf{G}$ for the restricted trait $j$, a Lagrange multiplier $(\lambda)$ to the vector $\mathbf{b}$, and a row with desired gains to $\mathbf{G}$ (Cunningham et al., 1970; Lin, 2005):

$$
\left[\begin{array}{ll}
\mathbf{P} & \mathbf{G}_{\mathbf{j}} \\
\mathbf{G}_{\mathbf{j}}^{\prime} & 0
\end{array}\right]\left[\begin{array}{l}
\mathbf{b} \\
\boldsymbol{\lambda}
\end{array}\right]=\left[\begin{array}{c}
\mathbf{G} \mathbf{v} \\
\frac{\Delta \mathbf{G}_{\mathbf{j}}}{\mathbf{i}} \sigma_{\mathbf{I}}
\end{array}\right]
$$

where $\mathbf{G}_{\mathbf{j}}$ is the $j$ th column of $\mathbf{G}$, corresponding to the restricted trait. Equation 3 is solved for $b$ and $\lambda$ using Equation 2, as for the unrestricted index. Because the standard deviation of the index $\left(\sigma_{I}=\sqrt{\mathbf{b}^{\prime} \mathbf{P b}}\right)$ depends on the solution of the selection index weights, iterations are needed to find the value of $\mathbf{G}_{\mathbf{j}}^{\prime} \mathbf{b}$, which yields the desired absolute response $\left(\Delta G_{j}\right)$ for trait $j$.

Total market economic response is maximized under the restriction that the covariance between index $(I)$ and the additive genotype for trait $j\left(\mathbf{G}_{\mathbf{j}}^{\prime} \mathbf{b}\right)$ equals $\frac{\Delta \mathbf{G}_{\mathbf{j}}}{\mathbf{i}} \sigma_{\mathrm{I}}$. Total market economic response $\left(\Delta \mathbf{H}_{\mathbf{E V}}\right)$ is predicted as:

$$
\Delta \mathbf{H}_{\mathbf{E V}}=\sum_{\mathbf{j}} \mathbf{E V}_{\mathbf{j}} \Delta \mathbf{G}_{\mathbf{j}}
$$

where $\mathrm{EV}_{j}$ is the market economic value for the $j$ th trait. Likewise, total nonmarket response $\left(\Delta \mathbf{H}_{\mathbf{N V}}\right)$ is predicted as:

$$
\Delta \mathbf{H}_{\mathrm{NV}}=\sum_{\mathbf{j}} \mathrm{NV}_{\mathrm{j}} \Delta \mathbf{G}_{\mathbf{j}}
$$

where $\mathrm{NV}_{j}$ is the nonmarket value for the $j$ th trait. Total selection response is then the sum of $\Delta \mathbf{H}_{\mathbf{E V}}$ and $\Delta \mathbf{H}_{\mathrm{NV}}$.

Restricting the Response in MY. With the response in MY restricted to a given PCTLOSS, the desired response for MY is

$$
(100-\mathbf{x}) \Delta \mathbf{G}_{\mathbf{E V}}
$$

where $\Delta G_{\mathrm{EV}}$ is selection response for MY using the breeding goal with EV only (0 PCTLOSS), $\mathbf{x}$ is a variable between 0 and 100, representing PCTLOSS (e.g., with 1 PCTLOSS, the selection response for MY is $99 \%$ of the selection response for MY based on the breeding goal with EV only). The covariance between the index and the additive genotype of $\mathbf{M Y}\left(\mathbf{G}_{\mathbf{M Y}}^{\prime} \mathbf{b}\right)$ should then be equal to

$$
\frac{(100-\mathbf{x}) \Delta \mathbf{G}_{\mathbf{E V}}}{\mathbf{i}} \sigma_{\mathbf{I}}
$$

With the studied traits and applied parameters (Table 1 ), the matrices of $\mathbf{G}$ and $\mathbf{P}$, and the vector $\mathbf{v}$ are 


$$
\begin{gathered}
\mathbf{G}=\left[\begin{array}{rrrr}
0.140 & -0.019 & -0.016 & 0 \\
-0.019 & 0.020 & 0.003 & 0 \\
-0.016 & 0.003 & 0.015 & 0 \\
0 & 0 & 0 & 0.020
\end{array}\right] \\
\mathbf{P}=\left[\begin{array}{rrrr}
0.079 & -0.009 & -0.009 & 0 \\
-0.009 & 0.020 & 0.002 & 0 \\
-0.009 & 0.002 & 0.017 & 0 \\
0 & 0 & 0 & 0.020
\end{array}\right] \mathbf{v}=\left[\begin{array}{r}
148 \\
163 \\
65 \\
-39
\end{array}\right]
\end{gathered}
$$

Equation 3, with the response for MY restricted to a given PCTLOSS (RMY index), can then be written as

$$
\begin{aligned}
& {\left[\begin{array}{rrrrr}
0.079 & -0.009 & -0.009 & 0 & 0.140 \\
-0.009 & 0.020 & 0.002 & 0 & -0.019 \\
-0.009 & 0.002 & 0.017 & 0 & -0.016 \\
0 & 0 & 0 & 0.020 & 0 \\
0.140 & -0.019 & -0.016 & 0 & 0
\end{array}\right]\left[\begin{array}{c}
\mathbf{b}_{\mathrm{MY}} \\
\mathbf{b}_{\mathrm{MR}} \\
\mathbf{b}_{\mathrm{CR}} \\
\mathbf{b}_{\mathrm{SB}} \\
\lambda_{\mathrm{MY}}
\end{array}\right]=} \\
& {\left[\begin{array}{r}
16.66 \\
0.74 \\
-0.83 \\
-0.78 \\
\frac{(100-\mathbf{x}) \Delta \mathbf{G}_{\mathbf{E v}}}{\mathbf{i}} \sigma_{\mathbf{I}}
\end{array}\right]}
\end{aligned}
$$

Iterations to the fifth decimal were used to find the value of $\mathbf{G}_{\mathbf{M Y}}^{\prime} \mathbf{b}$, which yielded the desired PCTLOSS. Solutions for $b$ and $\lambda$ were derived from Equation 2. In this case, $\lambda$ is the reduction in EV for MY to obtain a given PCTLOSS. Relative GV for MY, MR, CR, and $\mathrm{SB}$, yielding the desired response for MY, were derived based on the calculated $b$ values using Equation 6:

$$
\mathbf{G V}=\left(\mathbf{G}^{\prime} \mathbf{G}\right)^{-\mathbf{1}} \mathbf{G}^{\prime} \mathbf{P b} \text {. }
$$

The relative GV for $\mathrm{MY}$ is the original $\mathrm{EV}$, which is reduced corresponding to the loss in response in MY. The absolute GV for MR, CR, and SB were then derived by multiplying the EV (Table 1) by the ratio between the original EV for MY (Table 1) and the relative GV for MY. Finally, NV for MR, CR, and SB were derived as

$$
N V=G V-E V .
$$

Restricting the Response in Both MY and MR. Because the RMY index described above yielded a much higher response for MR than for $\mathrm{CR}, \mathrm{NV}$ were also derived using an index in which response in MY was restricted to a given PCTLOSS and MR was restricted to zero response (RMR index). The desired gain index theory with multiple restrictions is as presented by Lin (2005):

$$
\left[\begin{array}{rrr}
\mathbf{P} & \mathbf{G}_{\mathbf{1}} & \mathbf{G}_{\mathbf{2}} \\
\mathbf{G}_{\mathbf{1}} & 0 & 0 \\
\mathbf{G}_{\mathbf{2}} & 0 & 0
\end{array}\right]\left[\begin{array}{c}
\mathbf{b} \\
\lambda_{1} \\
\lambda_{2}
\end{array}\right]=\left[\begin{array}{r}
\mathbf{G a} \\
0 \\
\frac{\Delta \mathbf{G}_{\mathbf{2}}}{\mathbf{i}} \sigma_{\mathbf{I}}
\end{array}\right]
$$

where $G_{1}$ and $G_{2}$ are the columns of the $\mathbf{G}$ matrix for trait 1 restricted to 0 and for trait 2 restricted to a desired absolute response, and $\lambda_{1}$ and $\lambda_{2}$ are Lagrange multipliers. Below are the equations with MR restricted to 0 response and MY restricted to a given $(\mathbf{x})$ PCTLOSS:

$$
\begin{aligned}
& {\left[\begin{array}{rrrrrr}
0.079 & -0.009 & -0.009 & 0 & -0.019 & 0.140 \\
-0.009 & 0.020 & 0.002 & 0 & 0.020 & -0.019 \\
-0.009 & 0.002 & 0.017 & 0 & 0.003 & -0.016 \\
0 & 0 & 0 & 0.020 & 0 & 0 \\
-0.019 & 0.020 & 0.003 & 0 & 0 & 0 \\
0.140 & -0.019 & -0.016 & 0 & 0 & 0
\end{array}\right]\left[\begin{array}{c}
\mathbf{b}_{\mathbf{M Y}} \\
\mathbf{b}_{\mathbf{M R}} \\
\mathbf{b}_{\mathbf{C R}} \\
\mathbf{b}_{\mathbf{S B}} \\
\lambda_{\mathbf{M R}} \\
\lambda_{\mathbf{M Y}}
\end{array}\right]=}
\end{aligned}
$$

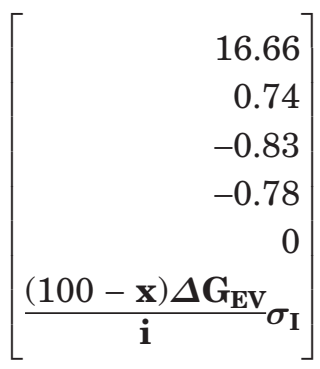

Restricting the Response in MY and the Relative Response Between MR and CR. The RREL index (response in MY restricted to a given PCTLOSS while simultaneously restricting the relative response between MR and CR) was applied to obtain a more equal response in MR and CR than when using the RMY index. The general equations for restricting a trait to an absolute desired response while simultaneously restricting the relative response between 2 other traits (Lin, 2005) are

$$
\left[\begin{array}{ccccc}
\mathbf{P} & \mathbf{G}_{\mathbf{1}} & \mathbf{G}_{\mathbf{2}} & 0 & \mathbf{G}_{\mathbf{3}} \\
\mathbf{G}_{\mathbf{1}} & 0 & 0 & -\mathbf{k}_{\mathbf{1}} & 0 \\
\mathbf{G}_{\mathbf{2}} & 0 & 0 & -\mathbf{k}_{\mathbf{2}} & 0 \\
0 & -\mathbf{k}_{\mathbf{1}} & -\mathbf{k}_{\mathbf{2}} & 0 & 0 \\
\mathbf{G}_{\mathbf{3}} & 0 & 0 & 0 & 0
\end{array}\right]\left[\begin{array}{l}
\mathbf{b} \\
\lambda_{1} \\
\lambda_{2} \\
\zeta \\
\lambda_{3}
\end{array}\right]=\left[\begin{array}{r}
\mathbf{G a} \\
0 \\
0 \\
0 \\
\frac{\Delta \mathbf{G}_{\mathbf{3}}}{\mathbf{i}} \boldsymbol{\sigma}_{\mathbf{I}}
\end{array}\right]
$$


where $G_{1}$ and $G_{2}$ are the columns of $\mathbf{G}$ for the 2 traits with the relative restricted response. $G_{3}$ is the column of the $\mathbf{G}$ matrix for the trait with a specified absolute desired response, $\mathbf{k}$ is a vector with proportional changes for traits 1 and 2, and $\lambda$ and $\zeta$ are Lagrange multipliers. With MY restricted to a given PCTLOSS and the (negative) response for CR restricted to 1.7 times the response for MR, the desired gain index is

$\left[\begin{array}{rrrrrrrr}0.079 & -0.009 & -0.009 & 0 & -0.019 & -0.016 & 0 & 0.140 \\ -0.009 & 0.020 & 0.002 & 0 & 0.020 & 0.003 & 0 & -0.019 \\ -0.009 & 0.002 & 0.017 & 0 & 0.003 & 0.015 & 0 & -0.016 \\ 0 & 0 & 0 & 0.020 & 0 & 0 & 0 & 0 \\ -0.019 & 0.020 & 0.003 & 0 & 0 & 0 & -1 & 0 \\ -0.016 & 0.003 & 0.015 & 0 & 0 & 0 & -1.7 & 0 \\ 0 & 0 & 0 & 0 & -1 & -1.7 & 0 & 0 \\ 0.140 & -0.019 & -0.016 & 0 & 0 & 0 & 0 & 0\end{array}\right]$

$$
\left[\begin{array}{c}
\mathbf{b}_{\mathrm{MY}} \\
\mathbf{b}_{\mathrm{MR}} \\
\mathbf{b}_{\mathbf{C R}} \\
\mathbf{b}_{\mathrm{SB}} \\
\lambda_{\mathrm{MR}} \\
\lambda_{\mathbf{C R}} \\
\zeta \\
\lambda_{\mathrm{MY}}
\end{array}\right]=\left[\begin{array}{r}
16.66 \\
0.74 \\
-0.83 \\
-0.78 \\
0 \\
0 \\
0 \\
\frac{(100-\mathbf{x}) \Delta \mathbf{G}_{\mathbf{E V}}}{\mathbf{i}} \sigma_{\mathbf{I}}
\end{array}\right]
$$

The 1.7:1 relationship between responses for CR and MR was chosen because it corresponds to responses for MR and CR at 0 PCTLOSS (the response for CR is more negative than the response for MR). To always have the most favorable response for MR, the response for $\mathrm{CR}$ was restricted to 1.7 times the selection response for MR when the response was negative, but when the response was positive, the response for MR was restricted to 1.7 times the selection response for CR.

\section{RESULTS}

\section{Restricting the Response in MY}

Figure 1 presents the NV for MR, CR, and SB as a function of the PCTLOSS in MY derived using the RMY index, with corresponding selection responses shown in Figure 2. It should be noted that for SB, the favorable change was negative (a decreased SB rate), but when presenting the results, the direction of the response for SB was changed such that a positive response was the favorable direction for all functional traits. The NV for MR, CR, and SB increased at the same rate with increasing PCTLOSS (Figure 1). However, the selection responses for the 3 traits did not change at the same

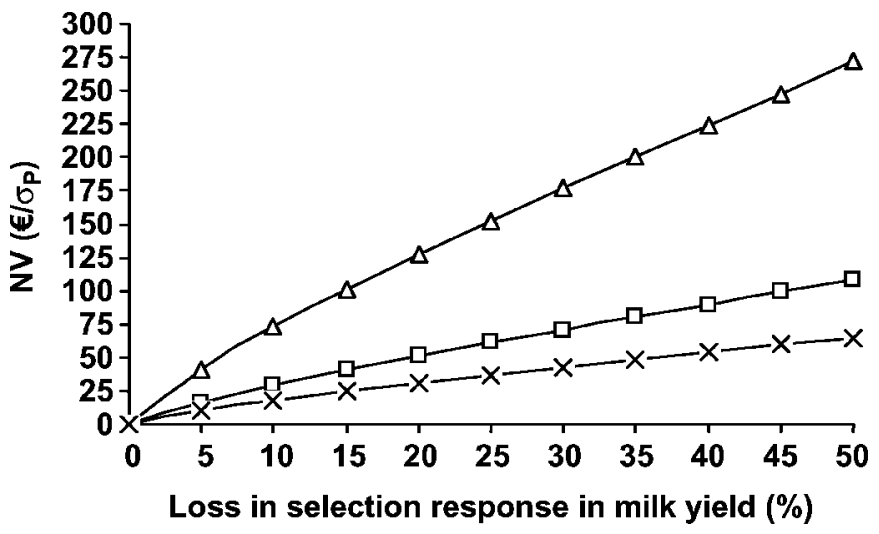

Figure 1. Nonmarket values (NV) for mastitis resistance $(\triangle)$, conception rate $(\square)$, and stillbirth $(\times)$ in euros per phenotypic standard deviation as a function of the percent loss in selection response for milk yield. Selection response for milk yield with 0 loss (corresponding to a breeding goal with market economic values only) is $141.0 €$.

rate (Figure 2A). The selection responses for MR, CR, and SB were $-0.23,-0.45$, and 0.13 with 0 PCTLOSS. In comparison, the selection responses with 5 PCTLOSS were $-0.07,-0.37$, and 0.16 for $\mathrm{MR}, \mathrm{CR}$, and $\mathrm{SB}$, respectively. The high response for MR compared with CR was due to the higher EV and heritability for MR. Similarly, a positive selection response for MR was obtained at approximately 7 PCTLOSS, whereas a loss of more than $37 \%$ in response in MY was needed to obtain a positive response for $\mathrm{CR}$. In addition, the percent reduction in total market economic response $\left(\Delta H_{\mathrm{EV}}\right)$ was lower than the PCTLOSS in MY (Figure 2B). For example, 5 PCTLOSS in MY reduced the total market economic response by only $0.7 \%$. Even though the total market economic response decreased with increasing PCTLOSS, the total selection response (market economic response + nonmarket response) was more than 15 PCTLOSS higher than the total market response because of the increased nonmarket response (Figure $2 \mathrm{~B})$. The increase in total nonmarket response was mainly due to the increased response for MR (Figure $2 \mathrm{~A})$.

\section{Restricting the Response in Both MY and MR}

If the only restriction made was that the response for MR should be zero, nearly 7 PCTLOSS in MY was obtained. Hence, the NV and selection responses using the RMR index are presented with 7 to 50 PCTLOSS (Figures 3 and 4). With increasing PCTLOSS, the NV for CR increased while the NV for MR decreased. The decreasing NV for MR can be explained by the fact that a lower NV was needed to keep the response for MR at zero, because of an indirect selection response as a result of the increased NV for CR. Using the RMR index, 

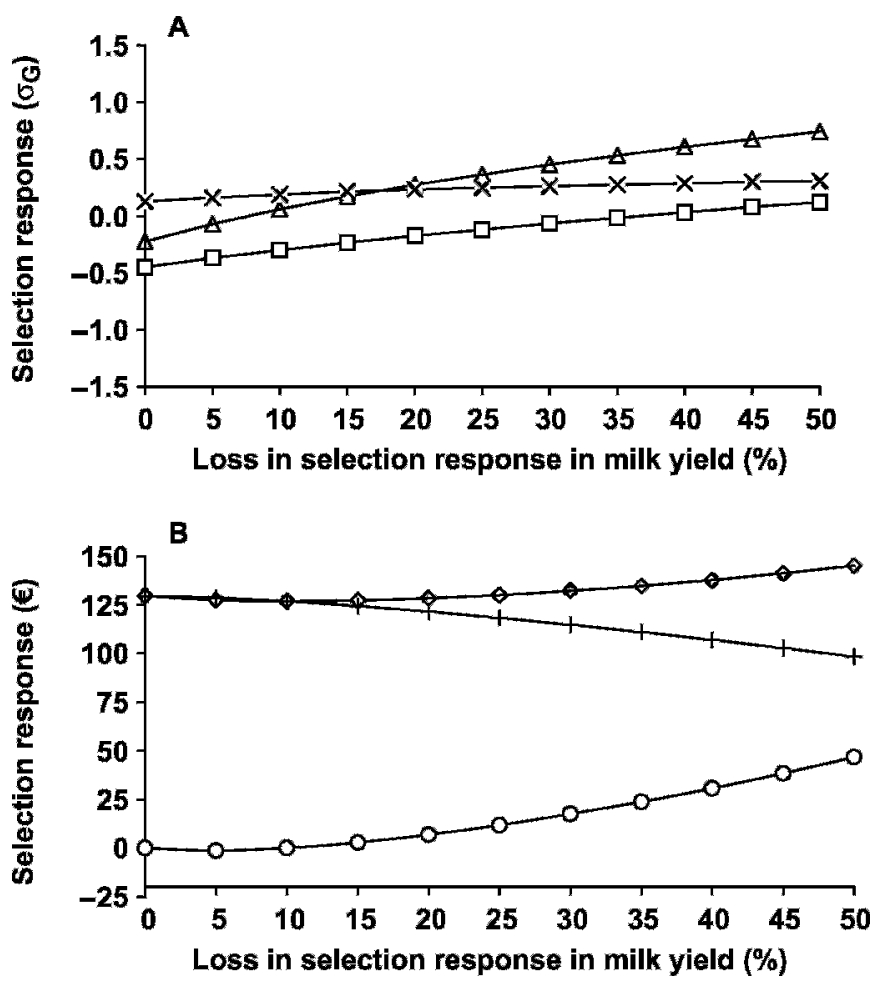

Figure 2. A) Selection response for mastitis resistance $(\triangle)$, conception rate $(\square)$, and stillbirth $(\times)$ in genetic standard deviation units as a function of the percent loss in response in milk yield; (B) total market economic response $(+)$, total nonmarket response $(\bigcirc)$, and total selection response $(\diamond$ : market economic response + nonmarket response) in euros as a function of the percent loss in selection response for milk yield.

the NV for MR was zero at a 25 PCTLOSS, because the indirect selection response through CR was sufficient to keep the response for MR at zero. This does not mean that there was no NV in improving the MR, but that the NV came indirectly through the NV for CR. Restricting the response in MR to zero while simultaneously restricting the MY to more than 25 PCTLOSS had no meaning, because at that point, the NV for CR was sufficient to acquire a response for both $\mathrm{CR}$ and $\mathrm{MR}$.

To attain a positive response for CR using the RMR index (Figure 4A), the PCTLOSS had to be at least 19\%. In comparison, a PCTLOSS of approximately $37 \%$ was needed to attain a positive response for CR using the restriction in MY only (RMY index, Figure 2A). The total nonmarket response was slightly negative up to an 11 PCTLOSS, after which it increased (Figure 4B). This was also reflected in the total selection response, which below an 11 PCTLOSS was slightly lower than the total market economic response. The negative nonmarket response was due to the unfavorable response for CR (Figure 4A). Above an 11 PCTLOSS, the nonmarket response for SB was high enough to obtain a positive

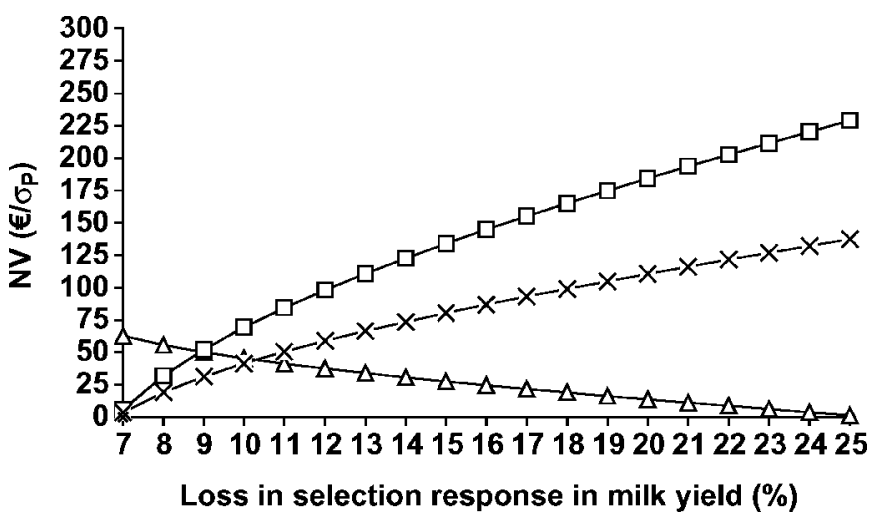

Figure 3. Nonmarket values (NV) for mastitis resistance $(\triangle)$, conception rate $(\square)$, and stillbirth $(\times)$ in euros per phenotypic standard deviation as a function of the percent loss in selection response in milk yield with mastitis resistance restricted to zero response.

total nonmarket response, even though the selection response for CR was unfavorable. The total market economic response was slightly lower for the RMR index than for the RMY index (i.e., $€ 126.5$ vs. $€ 126.9$ at a 10 PCTLOSS). The total nonmarket response was 11 PCTLOSS higher for the RMR index compared with the RMY index (i.e., €21.5 vs. €11.7 at a 25 PCTLOSS).

\section{Restricting the Response in MY and the Relative Response Between MR and CR}

Figures 5 and 6 show the NV and selection responses for the RREL index, in which the relative response between $\mathrm{CR}$ and $\mathrm{MR}$ was restricted. When the selection responses for $\mathrm{MR}$ and $\mathrm{CR}$ were negative (below a 16 PCTLOSS), the NV for CR increased substantially with increasing PCTLOSS, whereas the NV for MR increased only slightly (Figure 5). The increased NV for CR compared with MR was also reflected in the selection responses for the 2 traits (Figure 6A), with selection responses for $\mathrm{MR}$ and $\mathrm{CR}$ being more equal than when using the RMY (Figure 2A) or RMR (Figure 4A) indices. The selection response for SB increased up to 16 PCTLOSS, after which it decreased to a lower level. The decrease in response in SB was due to increasing selection emphasis on MR above a 16 PCTLOSS. The selection emphasis on MR was increased because above a 16 PCTLOSS (positive responses for MR and CR), the response for MR was restricted to 1.7 times the selection response for CR. But restricting the response for MR to 1.7 times the response for $\mathrm{CR}$ is not the same restriction as when restricting the response for CR to 1.7 times the response for MR (negative responses for MR and $\mathrm{CR}$ ). There were only small differences between the total market economic response and the total selection response (Figure 6B). This was because below a 16 

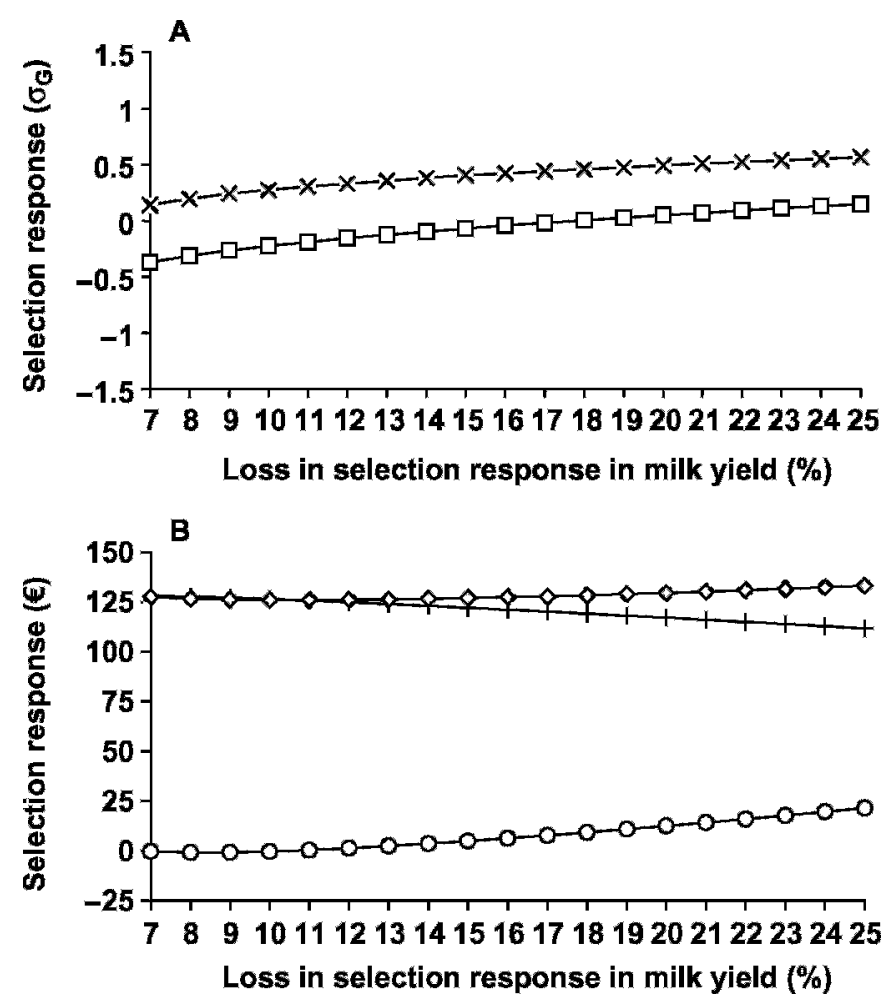

Figure 4. Selection response as a function of the percent loss in selection response in milk yield with mastitis resistance restricted to zero response. (A) Selection response for conception rate ( $\square$ ) and stillbirth $(x)$ in genetic standard deviation units; (B) total market economic response $(+)$, total nonmarket response $(\bigcirc)$, and total selection response $(\diamond:$ market economic response + nonmarket response $)$ in euros.

PCTLOSS, the total nonmarket response was almost constant owing to a low NV, especially for MR (Figure 5 ), and to the responses close to zero for both MR and CR (Figure 6A).

\section{DISCUSSION}

\section{Restricting the Response in MY to Improve Functional Traits}

In this study, the NV for multiple functional traits was derived by restricting the response in MY to a given PCTLOSS using the desired gain indices. The method presented is an extension of the method of Nielsen et al. (2005), who used selection index theory to derive the NV for a functional trait by predicting the loss in response in MY by adding a NV to the EV in the breeding goal. However, their method only allowed for derivation of the NV for one functional trait. By applying the desired gain indices to restrict the response in MY to a given PCTLOSS, as in this study, it was possible to derive the NV for several functional traits simultane-

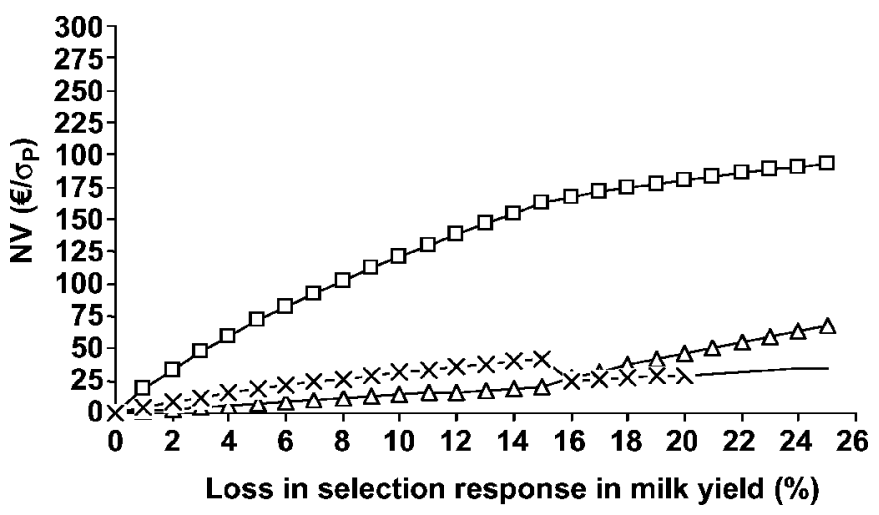

Figure 5. Nonmarket values (NV) for mastitis resistance $(\triangle)$, conception rate $(\square)$, and stillbirth $(x)$ in euros per phenotypic standard deviation as a function of the percent loss in selection response for milk yield. Up to $16 \%$ loss in response for milk yield, the relative response for conception rate is restricted to 1.7 times the selection response for mastitis resistance. After a $16 \%$ loss, the selection response for mastitis resistance is restricted to 1.7 times the selection response for conception rate.

ously. The NV for MR, CR, and SB using the RMY index (Figure 1) were all lower than the NV for the same traits and the same PCTLOSS derived one at a time by Nielsen et al. (2005). This was expected, because when the NV was derived simultaneously by using the RMY index, the PCTLOSS was distributed to each of the functional traits, compared with when the NV for traits were derived one at a time. Hence, when the NV were derived simultaneously using the RMY index, the selection response increased for all functional traits for a given PCTLOSS (Figure 2A), but when the NV were derived one at a time (Nielsen et al., 2005), the selection response increased mainly for the traits for which the NV were derived, because the NV for other traits were assumed to be zero. However, for the correlated traits, adding a NV to a single trait influenced the selection response for the other traits (Nielsen et al., 2005).

\section{Restricting the Response in Both MY and Functional Traits}

The RMR and RREL indices were applied because the selection response was much lower for CR than for MR when the NV was derived using the RMY index (restriction in MY only). Applying the RMR index increased the response for CR for a given PCTLOSS (Figure 4A) compared with the RMY index (Figure 2A). With respect to sustainable development, it might be better to apply the RMR or RREL indices than the RMY index, because responses for the traits can be differentiated according to the desired development. This would especially be an advantage for a trait with a very unfavorable response or for a trait at an unac- 

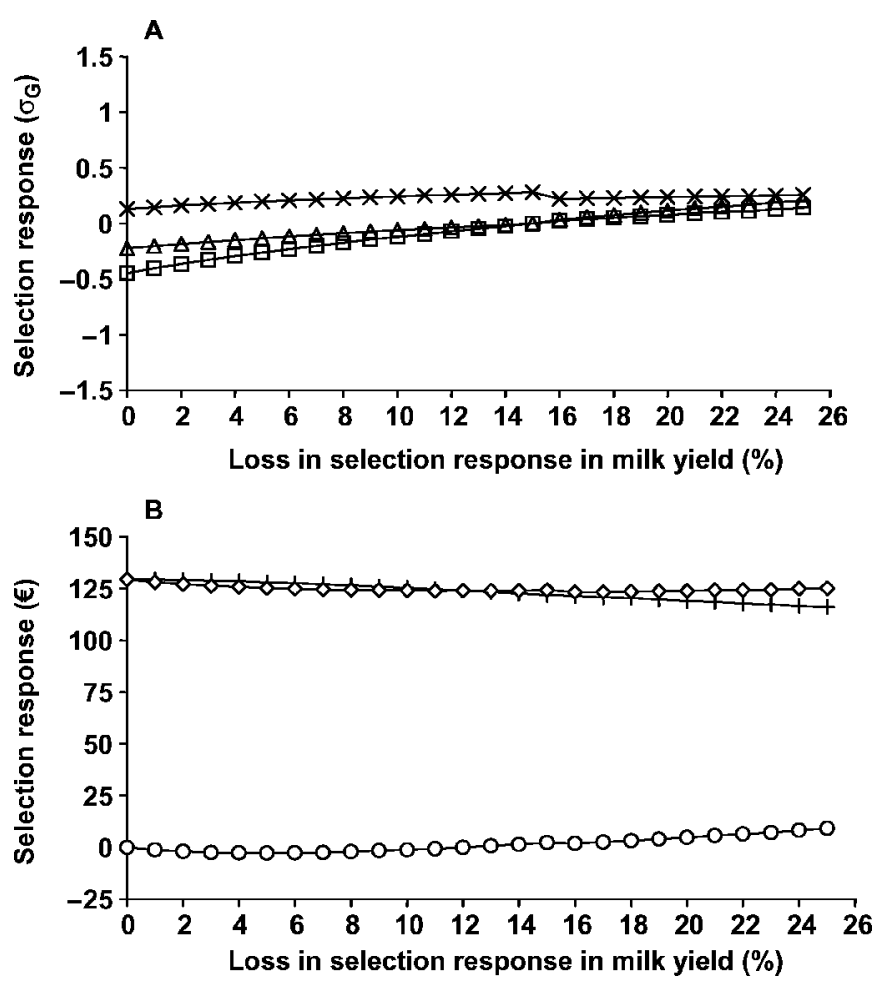

Figure 6. Selection response as a function of the percent loss in selection response in milk yield. Up to a $16 \%$ loss in response in milk yield, the relative response for conception rate is restricted to 1.7 times the selection response for mastitis resistance. After a $16 \%$ loss, the selection response for mastitis resistance is restricted to 1.7 times the selection response for conception rate. (A) Selection response for mastitis resistance $(\triangle)$, conception rate $(\square)$, and stillbirth $(\times)$ in genetic standard deviation units; (B) total market economic response $(+)$, total nonmarket response $(\bigcirc)$, and total selection response $(\diamond$ : market economic response + nonmarket response) in euros.

ceptably low level. However, the disadvantage of using the RMR index, compared with the RMY index, is that one should be willing to lose at least $7 \%$ in the selection response for MY (the loss when the only restriction is that the response for MR is zero). In addition, the total market economic response was lower when using the RMR and RREL indices (Figures 4B and 6B) compared with the RMY index (Figure 2B). But after approximately 11 PCTLOSS, the total nonmarket response was higher for the RMR index than for the RMY index. Restrictions in both MY and functional traits make it possible to obtain the desired responses for functional traits, but the total market economic response will always be lower than when the restriction is solely on MY. On the other hand, if one is willing to lose more than $11 \%$ in response in MY, the total nonmarket response and the total response will be higher when using the RMR index than when using the RMY index. On the other hand, by using the RREL index there is little to gain in the total nonmarket response. Thus, the choice of a breeding goal aiming at sustainable development depends on how much one is willing to lose in response in MY and on the responses for individual traits. Below an 11 PCTLOSS, the total nonmarket response is lower for the RMR index compared with the RMY index, but above an 11 PCTLOSS, the opposite is true.

In this study, the total selection response was valued by both the EV and the NV (Equations 4 and 5). As discussed by Olesen et al. (2000) and Nielsen et al. (2005), a breeding goal including EV may be too narrowminded, because the EV may represent only economic aspects of the current market economy. In contrast, the NV represents a wider perspective, including social aspects. Furthermore, goals including the NV may, in some cases, be considered as more far-sighted breeding goals, because these may aim at improving functional traits beyond those known as economically profitable in the current market economy. Values that are NV today may therefore become $\mathrm{EV}$ in a future market economy in which, for example, functional traits gain a higher EV than today. However, it still remains to be decided whether farmers should assume all the cost of improving a socially important trait or whether members of a society should cover some of the losses (Nielsen et al., 2005).

The methods presented in this study were based on the trade-off between selection responses for production and functional traits. Most breeding companies (in particular, breeding companies for dairy cattle) select animals on total merit indices based on market EV. Therefore, a possible next step in the development of the approach could be to look at the trade-off between the total market economic response (valued by EV only) vs. the response for functional traits.

Not all combinations of restrictions in the different traits are possible. For example, with a 1 PCTLOSS in the selection response for MY, the selection response for MR will necessarily be negative, and can thus not be restricted to zero. To explore possible restrictions, the selection response surface of the traits can be created by varying the GV for each of the traits. However, with an increasing number of traits in the breeding goal, such multidimensional graphs become increasingly complicated to interpret.

The selection responses for traits depend on assumptions about the structure of the breeding plan as well as genetic, phenotypic, and economic parameters. Instead of adding a NV to the EV for functional traits, an increased selection response could also be obtained by changing the structure of the breeding plan, such as by increasing the progeny group size. However, increasing the number of daughters per sire would probably lead to reduced selection intensity and thereby pos- 
sibly reduce the total market economic response. Moreover, deciding on the size of progeny groups is part of the optimization of the breeding plan, which should be performed based on both the EV and the NV. In this study, a progeny group size of 100 daughters was assumed to be optimal.

\section{Methods to Define Sustainable Breeding Goals}

Olesen et al. (1999) suggested methods that could possibly be used to derive the NV. Among others, these are desired gain indices and methods based on consumers' willingness to pay for a given product. Olesen et al. (2005) performed a market study to estimate consumers' willingness to pay for the improved welfare of fish. People were asked to choose between types of salmon filets labeled according to differences in the production system (conventional or ecological). The study showed that people were willing to pay $€ 2$ more per kilogram for salmon filets produced under ecological conditions compared with conventionally produced salmon filets. Animal welfare is a public good, which is only partly valued or traded in the market (Olesen et al., 2005). If products can be labeled in the stores as being produced with a higher level of animal welfare, part of the value of improved welfare can be estimated, as shown in the study by Olesen et al. (2005). This also means that if it is possible to label products in the future, the value of improved animal welfare will no longer be a NV but an EV, because the improved welfare of the fish would then be reflected in the market price. However, the market price of a product being labeled as produced with a higher level of animal welfare does not necessarily reflect the total value of improved animal welfare, because people not eating fish also benefit from improved welfare (Olesen et al., 2005). Additionally, the government might introduce taxes or subsidies, making an increase in the NV of a trait possible without a change in the market price of products. Contingent valuation methods can possibly be applied to reveal people's willingness to support governmental legislation or subsidies through taxes (Olesen et al., 2005).

Methods based on selection index theory (as in the current method) have the advantage that they integrate the concept of sustainability into classical breeding methodology, which might appeal to farmers and breeding companies. Kanis et al. (2005) used so-called retrospective selection indices to define sustainable breeding goals for pig breeding programs. Their method was based on exploring the selection response surface for traits by increasing the GV for traits in the breeding goal that were likely to have a NV. Based on the selection response surface, breeding companies would then be able to choose the GV corresponding to the response that they found the most sustainable or acceptable.

Communication is important when considering sustainability, and animal breeders and scientists should take into account the opinions of members of society when defining breeding goals (Gamborg and Sandøe, 2005). It might be easier to communicate based on selection response instead of GV (Kanis et al., 2005), because the GV in itself does not provide much information about the actual selection emphasis on a given trait. Using the method presented in this study, it is possible to describe the response in functional traits directly, based on how much farmers and breeding companies are willing to lose with respect to production traits. When considering sustainability, animal breeders need to negotiate trade-offs (Gamborg and Sandøe, 2005). The present method can be used to address one of these trade-offs-namely, increases in productivity and efficiency (MY and CR) - while increasing animal health and welfare by decreasing the SB rate and incidences of mastitis.

One of the disadvantages of the current method is that both the choice of the level of loss in response in MY and the choice of restriction:desired gain index is subjective. Levels of total nonmarket response, total market economic response, and total response can be helpful in choosing a restriction and level of loss in response in MY, but this remains a subjective judgment. In addition, by applying the RMR index, one assumes that the current level of MR in the population is acceptable. This implies that there is a need to provide an objective basis and the information needed for decision makers to choose the restriction and the level of acceptable losses when defining the most suitable breeding goal for sustainable development. When deciding on the acceptable level of loss in production and on the choice of restriction, breeding organizations should involve policy makers and consumers to include their concerns about animal production. Methods based on quantifying people's willingness to pay for products from animals with better welfare might be used to predict future trends and thereby give some indications about the level of trade-off in production and functional traits. Another disadvantage with the current method is that only one production trait can be considered. It might be relevant to extend the method to consider more production traits, because some breeding goals for dairy cattle include both MY and beef production (Mark, 2004). This means that there is a need to show how losses in response in production should be distributed to each of the production traits in the breeding goal. However, extending the method to include more production traits might also further complicate the choice of level of accepted loss and choice of restriction. 
Although the method presented here provides a useful tool in the process toward developing breeding goals for sustainable production, there is still a need both for further development of the current method and for further development of methods to derive NV.

This study illustrates the current method by using an example from dairy cattle breeding with 3 functional traits only. The method can easily be extended to more functional traits, as in most dairy cattle breeding goals, but adding more functional traits may further complicate the choice of level of accepted loss and the choice of restriction. The method can also be applied to other livestock species such as pigs, poultry, or fish by changing the index equations according to the structure of their breeding plan. However, most breeding goals for poultry and fish are already based on desired gain indices. The EV derived using the desired gain indices is not aimed at maximizing profits, and it yields a lower market economic response than the EV based on profit equations (Gibson and Kennedy, 1990). Therefore, when possible, the EV should be derived using profit equations or bioeconomic models, and the desired gain indices or other methods, such as the one presented in this paper, should be applied to derive sustainable breeding goals.

As already indicated, a desired gain index will result in less total market economic response than an index based on the EV derived using a profit equation (Gibson and Kennedy, 1990). Therefore, desired gain indices cannot be recommended as a tool for derivation of $\mathrm{EV}$. However, as shown in this study and as discussed by Brascamp (1984) and Christensen (1998), the desired gain indices can be used in combination with the EV based on maximizing the farmer's profit for the definition of breeding goals. By applying the desired gain indices, the derivation of a sustainable breeding goal is a 3-step procedure. First, the EV should be derived based on profit equations or a bioeconomic model aiming at maximizing farmers' profit. Second, the breeding program should be optimized and the selection response for all traits predicted based on the EV. Finally, the $\mathrm{NV}$ should be derived and added to the EV for traits with an unacceptable selection response, and the breeding program should be reoptimized.

Currently, most breeding goals for dairy cattle are trending toward including both MY and several functional traits (Mark, 2004; Miglior et al., 2005). However, as shown in this study, the EV of a trait (which is expected to maximize the profit of the farmer) might not be sufficient to avoid deterioration of the trait. By implementing the NV in the breeding goal, functional traits can be given appropriate weight to avoid further deterioration because of intensive selection for MY. Therefore, this method can be used by breeding compa- nies to increase the response for traits that are at a critically low level, or to favor traits they think will have a higher EV in the future than at present. Further, this method may also improve the competitive position of their breeding companies because farmers tend to choose sires not only based on their total merit index estimated based on the EV, but also on performance for individual traits. A low-performing sire for a given trait compared with the performance of sires from another breeding company is not necessarily compensated for by high performance in other traits (de Vries, 1989).

\section{ACKNOWLEDGMENTS}

The authors would like to thank Theo Meuwissen for helpful ideas concerning this study and Bjarne Gjerde for comments on the manuscript. The study was financed by The Research Council of Norway, grant no. 143045/140 (Future Animal Breeding Goals).

\section{REFERENCES}

Brascamp, E. W. 1984. Selection indices with constraints. Anim. Breed. Abstr. 52:645-654.

Brascamp, E. W., C. Smith, and D. R. Guy. 1985. Derivation of economic weights from profit equations. Anim. Prod. 40:175-180.

Christensen, L. G. 1998. Future market and consumer-oriented breeding goals. Acta Agric. Scand. Anim. Sci. (Suppl. 28):45-53.

Code-EFABAR. 2004. Code of good practice for farm animal breeding organisations. Version 2005. Working Party Code-EFABAR. http://www.sefabar.org/code-efabar. Accessed Nov. 22, 2005.

Cunningham, E. P., R. A. Moen, and T. Gjedrem. 1970. Restriction of selection indexes. Biometrics 26:67-74.

de Vries, A. G. 1989. A method to incorporate competitive position in the breeding goal. Anim. Prod. 48:221-227.

Gamborg, C., and P. Sandøe. 2005. Sustainability in farm animal breeding: A review. Livest. Prod. Sci. 92:221-231.

Gibson, J. P., and B. W. Kennedy. 1990. The use of constrained selection indexes in breeding for economic merit. Theor. Appl. Genet. 80:801-805.

Hazel, L. N. 1943. The genetic basis for constructing selection indexes. Genetics 28:476-490.

Kanis, E., K. H. De Greef, A. Hiemstra, and J. A. M. van Arendonk. 2005. Breeding for societally important traits in pigs. J. Anim. Sci. 83:948-957.

Lin, C. Y. 2005. A simultaneous procedure for deriving selection indexes with multiple restrictions. J. Anim. Sci. 83:531-536.

Mark, T. 2004. Applied genetic evaluations for production and functional traits in dairy cattle. J. Dairy Sci. 87:2641-2652.

Miglior, F., B. L. Muir, and B. J. Van Doormaal. 2005. Selection indices in Holstein cattle of various countries. J. Dairy Sci. 88:1255-1263.

Nielsen, H. M. 2004. Economic values for production and functional traits in dairy cattle breeding goals derived by stochastic simulation. $\mathrm{PhD}$ thesis, Royal Veterinary and Agricultural University, Copenhagen, Denmark.

Nielsen, H. M., L. G. Christensen, and A. F. Groen. 2005. Derivation of sustainable breeding goals for dairy cattle using selection index theory. J. Dairy Sci. 88:1882-1890.

Olesen, I., F. Alfnes, M. Røra, S. Navrud, and K. Kolstad. 2005. Economic values of fish welfare in breeding goals, and application of market experiments. Pages 348-349 in Aquaculture Europe 2005: "Optimising the Future," Trondheim, Norway. European 
Aquaculture Society Special Publication no. 35. European Aquaculture Society, Oostende, Belgium.

Olesen, I., B. Gjerde, and A. F. Groen. 1999. Methodology for deriving non-market trait values in animal breeding goals for sustainable production systems. Pages 13-21 in Proc. Int. Workshop on EU Concerted Action on Genetic Improvement of Functional Traits in Cattle (GIFT). Interbull Bulletin no. 23. Interbull, Wageningen, The Netherlands.
Olesen, I., A. F. Groen, and B. Gjerde. 2000. Definition of animal breeding goals for sustainable production systems. J. Anim. Sci. 78:570-582.

Sandøe, P., B. L. Nielsen, L. G. Christensen, and P. Sørensen. 1999. Staying good while playing God-The ethics of breeding farm animals. Anim. Welf. 8:313-328.

Sørensen, M. K. 1999. Stochastic simulation of breeding schemes for dairy cattle. Report. Danish Institute of Agricultural Sciences, Tjele, Denmark. (in Danish with English summary) 